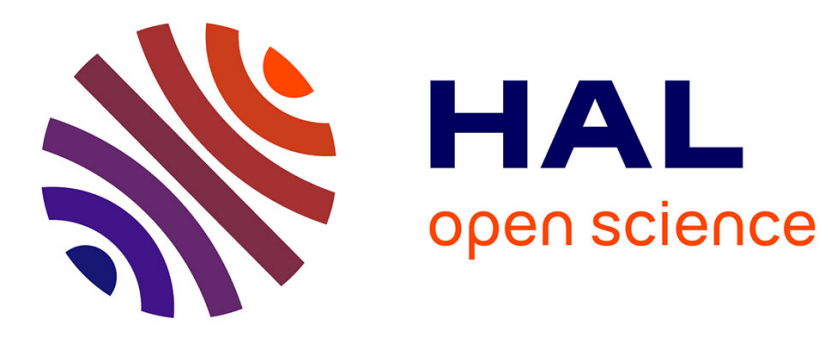

\title{
Surface tension of functionalized MWCNT-based nanofluids in water and commercial propylene-glycol mixture
}

Nawal Berrada, Samah Hamze, Alexandre Desforges, Jaafar Ghanbaja, Jérôme Gleize, Thierry Mare, Brigitte Vigolo, Patrice Estellé

\section{To cite this version:}

Nawal Berrada, Samah Hamze, Alexandre Desforges, Jaafar Ghanbaja, Jérôme Gleize, et al.. Surface tension of functionalized MWCNT-based nanofluids in water and commercial propylene-glycol mixture. Journal of Molecular Liquids, 2019, 293, pp.111473. 10.1016/j.molliq.2019.111473 . hal-02265963

HAL Id: hal-02265963

https://hal-univ-rennes1.archives-ouvertes.fr/hal-02265963

Submitted on 23 Sep 2019

HAL is a multi-disciplinary open access archive for the deposit and dissemination of scientific research documents, whether they are published or not. The documents may come from teaching and research institutions in France or abroad, or from public or private research centers.
L'archive ouverte pluridisciplinaire HAL, est destinée au dépôt et à la diffusion de documents scientifiques de niveau recherche, publiés ou non, émanant des établissements d'enseignement et de recherche français ou étrangers, des laboratoires publics ou privés. 
Surface tension of functionalized MWCNT-based nanofluids in water and commercial propylene-glycol mixture

Nawal Berrada ${ }^{1}$, Samah Hamze $^{2}$, Alexandre Desforges ${ }^{1}$, Jaafar Ghanbaja ${ }^{1}$, Jérôme Gleize ${ }^{3}$, Thierry Maré2 Brigitte Vigolo $^{1}$, Patrice Estellé ${ }^{2, *}$

${ }^{1}$ Université de Lorraine, CNRS, Institut Jean Lamour UMR7198, F-54000 Nancy, France

${ }^{2}$ Université de Rennes, LGCGM EA3913, F-35000 Rennes, France

${ }^{3}$ Université de Lorraine, Laboratoire de Chimie et Physique Approche Multi-échelles des Milieux Complexes EA 4632, F-57000 Metz, France

\title{
* Corresponding author: patrice.estelle@univ-rennes1.fr
}

\begin{abstract}
:
Nanofluids which consist of the addition of nanoparticles in a base fluid are envisaged for a large domain of applications. For many of them, the surface tension (ST) behavior of the prepared nanofluids is a key parameter to exploit their thermophysical properties. Due to their remarkable properties, carbon nanotubes (CNTs) are commonly used to develop nanofluids. However, the evolution of the ST of CNT-based nanofluids is still far from being understood and predictable. In the present work, two base fluids were used: water and a commercial mixture of propyleneglycol/water (around 40:60 wt.\%). The impact of the used multi-walled CNT (MWCNT) addition, MWCNT concentration (0.001 and $0.1 \mathrm{wt} . \%)$ and temperature variation (273.15-333.15 K) on the density and ST evolution for the two kinds of CNT-based nanofluids is studied. The
\end{abstract}


chemically modified MWCNTs are assumed to bear both localized hydrophilic areas due to grafted functional groups and remaining hydrophobic surfaces. The found difference in ST behavior between the two types of nanofluids is explained in light with the involved interfaces in each nanofluid. ST evolution is found to strongly depend on the CNT surface properties.

\section{Keywords: MWCNT, nanofluids, chemical modification, surface tension, effect of concentration and base fluid}

\section{Introduction}

Nanofluids, a very simple product of the emerging world of nanotechnology, are suspensions of nanoparticles (1-100 nm in size) in conventional base fluids such as water, glycols, or oils. Since their introduction in the early 1990s, they have been the subject of many research works. The nanoparticles are typically made of metals, carbides, oxides, or carbon-based materials. As compared to the pure base fluids, this innovative class of heat transfer fluids exhibits much higher thermophysical properties including increase in thermal conductivity, high single-phase and boiling heat transfer coefficients, and enhanced critical heat flux. Due to their unique properties, nanofluids can be used in various applications including micro-channel cooling within integrated circuits, biomedical therapies and diagnosis, manufacturing procedures, chemical and pharmaceutical processes, engine and electronic device cooling, concentrating solar energy, nuclear reactors, etc... [1]. In particular, the liquid-gas surface tension plays a major role in applications related to boiling and condensation phenomena, and other processes involving heat transfer [2]. However, how surface tension (ST) may change with particle 
parameters such as concentration, size and shape, nature of the used nanoparticles, and also with the type of base fluids and surfactant is an emerging topic. Some recent efforts have been devoted towards defining and measuring the surface tension of nanofluids as reviewed in ref. [2]. In addition, as nanoparticles suspended in the base fluid, carbon nanotubes (CNTs) have been widely analysed, but the properties of CNT-based nanofluids such as the contact angle of sessile droplets [3] and the effective gas-liquid surface tension of these nanofluids have been rarely investigated. Main results from the open literature are described in the following. Xue et al. [4] studied the ST of acid-treated CNTs dispersed in water. Experiments were performed from the bubble pressure method for a nanotube volume concentration of $1 \mathrm{vol} . \%$. They showed that the presence of CNTs in water increased the ST of water up to $13.5 \%$ and the ST of nanofluid decreased with rising temperature from 298.15 to $343.15 \mathrm{~K}$. Tanvir and Qiao [5] exploited the pendant drop method to measure the ST of multi-walled CNTs (MWCNTs) dispersed in water or ethanol. The measurements were done at ambient temperature and the studied weight concentration range was between 0.1 and $10 \mathrm{wt} \%$. They found that, for ethanol-based nanofluids, the ST decreased slightly up to $2 \mathrm{wt} . \%$, then it was increased with nanoparticle loading. For water-based nanofluids, an increase in ST with the mass content of CNTs was thus reported. Effect of sodium dodecyl benzene sulfonate (SDBS) surfactant addition and chemical treatment of single-walled carbon nanotubes SWCNTs dispersed in water and ST of resulting nanofluids were investigated by Murshed et al. [6] and Kumar and Milanova [7] for a concentration of 0.1 vol. $\%$. The authors showed that for SWCNT-based nanofluids, in a low surfactant concentration domain, ST did not vary with surfactant concentration. However, above a critical value of surfactant concentration, ST decreased because of the excess of surfactant in water. Interestingly, decrease in nanofluid ST with surfactant concentration was correlated to an 
observed increase in heat flux during pool boiling. Study of ST of functionalized MWCNT-based nanofluids was reported by Karthikeyan et al. [8]. Ethanol and water were used as base fluids. ST properties were obtained at ambient temperature with the pendant drop method. They highlighted that ST of well-stable nanofluids started to increase from a critical concentration value of $500 \mathrm{ppm}$ with ethanol, and did not depend on the MWCNT concentration for water as base fluid. In another work, Soleimani et al. [9] investigated the ST of distilled water-based nanofluids where different concentrations of MWCNTs. From 0.05 to 0.5 wt.\% of MWCNTs were dispersed by using sodium dodecyl sulfate (SDS) as a surfactant. They used the pendant drop method for their measurements, and they obtained that the ST increased steadily of up to 0.3 wt. $\%$ then it decreased. Ershadi et al. [10] prepared $0.2 \mathrm{wt} . \%$ of $\mathrm{MWCNT} \mathrm{SiO}_{2}$ nanohybridbased nanofluids with water as base fluid. They investigated the effect of nanofluids on the wettability of carbonate and sandstone substrates by measuring interfacial tension and contact angle. Their results showed that the nanofluid could significantly decrease the ST and change the wettability of the rock from oil-wet to water-wet conditions. A decreasing in ST was also obtained by AfzaliTabar et al. [11] with $\mathrm{MWCNT} / \mathrm{SiO}_{2}, \mathrm{SWCNT} / \mathrm{SiO}_{2}$, and activated carbon/ $/ \mathrm{SiO}_{2}$ dispersed in distilled water. They found a better ability of $\mathrm{MWCNT} / \mathrm{SiO}_{2}$ nano hybrid for decreasing the ST in comparison to the two other samples.

In summary, based on the literature review, it is difficult to predict how ST can change with addition of CNTs. Also, it is not clear whether the ST will increase or decrease with nanotube content with respect to the type of base fluid and what mechanisms are responsible for the observed behaviors. An attempt to give some highlights in this field has motivated the present research. So, the main objective of this work is to determine the effect of CNT addition and temperature on the ST of water-based nanofluids. Functionalized MWCNTs were dispersed in 
two different heat transfer water-based fluids, pure distilled water and Tyfocor® LS - a commercial fluid composed of propylene-glycol/water (around 40:60 wt.\%), up to now only used with nanofluids in solar collector absorber plate with microchannels [12] - respectively. The pendant drop method is used for the ST measurements and two distinct weight concentrations of functionalized MWCNTs are considered, 0.001 and $0.1 \mathrm{wt} . \%$, respectively. In the present study, the complete characterization of the raw and the chemically modified MWCNTs by using thermogravimetric analysis (TGA), transmission electron microscopy (TEM) and Raman spectroscopy is performed. Then, dispersion in the two used base fluids is analyzed by means of optical microscopy and zeta potential techniques. Therefore, the density of base fluids and nanofluids is evaluated. Finally, influence of the nature of the base fluid, the nanotube concentration and the applied temperature on the ST evolution is finally discussed in the light of the involved interfaces in each nanofluid.

\section{Materials and methods}

Raw MWCNT sample. The raw MWCNTs $\left(\right.$ Nanocyl $^{\mathrm{TM}}$ NC7000, average inner diameter $~ 9.2$ nm, average length: $1.5 \mu \mathrm{m}$ [13] were purchased from Nanocyl S.A. (Belgium). They were synthesized by catalytic chemical vapor deposition (CCVD) by using iron and cobalt as catalysts deposited on an alumina substrate. The raw MWCNTs are referred to as r-MWCNT.

Chemicals. Potassium permanganate $\left(\mathrm{KMnO}_{4}\right)$ and sodium sulfite $(\mathrm{Na} 2 \mathrm{SO} 3)$ were obtained from Alfa Aesar (Germany), sodium hydroxide $(\mathrm{NaOH})$ from VWR International (USA), and nitric acid $\left(\mathrm{HNO}_{3}\right) 65 \%$ was acquired from Honeywell Fluka International (USA). Sulfuric acid $\left(\mathrm{H}_{2} \mathrm{SO}_{4}\right)$ 95-97 \% and hydrochloric acid $(\mathrm{HCl}) 37 \%$ were both purchased from Sigma Aldrich (Germany). 
Chemical treatments. The carried-out purification is a one-pot gas-phase treatment [14]. Briefly, the raw MWCNTs $(\sim 300 \mathrm{mg})$ were placed in a silica crucible and introduced in a tubular oven. First, in order to remove air, the sample was vented by the carrier gas, nitrogen. The sample was then heated at $10 \mathrm{~K} / \mathrm{min}$ up to $1273.15 \mathrm{~K}$ under a stream of chlorine at a flow-rate of 200 $\mathrm{mL} / \mathrm{min}$. After $2 \mathrm{~h}$ at $1273.15 \mathrm{~K}$ under chlorine, the MWCNT powder was maintained at 1273.15 $\mathrm{K}$ for 2 additional hours under nitrogen before natural cooling.

After this treatment, the purified MWCNTs (referred to p-MWCNT) are ready to be used. pMWCNT was functionalized using a $\mathrm{KMnO}_{4} / \mathrm{NaOH}$ (1:1) mixture [15]. About $100 \mathrm{mg}$ of purified MWCNTs were put in $60 \mathrm{~mL}$ of $\mathrm{KMnO}_{4} 0.2 \mathrm{M}$ and $60 \mathrm{~mL}$ of $\mathrm{NaOH} 0.2 \mathrm{M}$ in a round bottom flask equipped with a condenser, and heated with an oil bath until refluxing maintained for $40 \mathrm{~min}$. The solution was then cooled down to room temperature. The excess of $\mathrm{KMnO}_{4}$ was reduced with $3 \mathrm{~g}$ of $\mathrm{Na}_{2} \mathrm{SO}_{3}$ in an acid media ( $35 \mathrm{~mL}$ of $\mathrm{H}_{2} \mathrm{SO}_{4} 1 \mathrm{M}$ ). Then, the mixture was filtered on a membrane (Merck Millipore, $0.45 \mu \mathrm{m}$ ). Firstly, it was washed with $\mathrm{HCl} 4 \mathrm{~N}$ then with distilled water until neutral $\mathrm{pH}$ and dried overnight at $323.15 \mathrm{~K}$. The functionalized MWCNTs thus obtained are denoted f-MWCNT in the following.

Nanofluid preparation. After purification and functionalization of MWCNTs, nanofluids were prepared from the two step-method by dispersing the chosen weight of f-MWCNT in either distilled water (DW) or water-propylene glycol-based solvent namely Tyfocor® LS (referred to Tyfocor in the following (figures and text) for sake of clarity). Two distinct concentrations of fMWCNT were selected to investigate the effect of nanoparticle content on the ST of nanofluids: 0.1 and 0.001 wt.\%. The solutions were sonicated using an ultrasonic probe Vibra cell 75042 during $1 \mathrm{~h}$ with a power of $125 \mathrm{~W}$ with a pulse mode: 2 sec-ON/1 sec-OFF under controlled temperature. 


\section{Characterization techniques.}

Thermogravimetric analysis (TGA) was performed with a Setaram Setsys evolution 1750. About $5 \mathrm{mg}$ of MWCNT powder loaded in a platinum crucible was transferred in the chamber where, after a purging stage, temperature was increased from room temperature to $1173.15 \mathrm{~K}$ with a ramp of $5 \mathrm{~K} / \mathrm{min}$. A $20 \mathrm{~mL} / \mathrm{min}$-flow rate of dry air or helium was used as a carrier gas. The metallic impurity content was determined by using the remaining weight after the combustion of the carbon species leading to a rapid decrease in weight.

Transmission electron microscopy (TEM) observations were carried out using a JEM-ARM $200 \mathrm{~F}$ apparatus at an accelerating voltage of $80 \mathrm{kV}$. The samples were prepared by dispersing a small amount of CNTs in ethanol using a sonication bath for a few minutes. A drop of the solution was deposited on a holey carbon grid (200 mesh size). Being aware that TEM is a local technique, about 30 images were taken at different areas for each sample to guarantee a statistical analysis.

Raman spectroscopy was carried out by a LabRAM HR 800 micro-Raman spectrometer using a red incident laser $(\lambda=632.8 \mathrm{~nm})$ and an x50 objective. A drop of the raw or treated MWCNTs dispersed in ethanol was deposited on a glass slide. The recorded number of spectra for each sample was at least 3 and could be higher according to the dispersion of the results. After subtraction of the baseline, the height of the $\mathrm{D}$ band was divided by that of the $\mathrm{G}$ band to calculate the $\mathrm{I}_{\mathrm{D}} / \mathrm{I}_{\mathrm{G}}$ intensity ratio. The error bars were determined from the standard deviation to the calculated $\mathrm{I}_{\mathrm{D}} / \mathrm{I}_{\mathrm{G}}$ average.

Optical microscopy was performed by an optical microscope Olympus TH4-200 BXS1 using an x10 objective. For the observations, a drop of a MWCNT solution was deposited on a glass slide recovered by a coverslip ( $22 \mathrm{~mm}$ x $22 \mathrm{~mm})$. 
A Malvern Zetasizer was used to measure the zeta potential of the functionalized MWCNTs dispersed in water.

Density measurements of base fluids and nanofluids were performed with a Rudolph Research Analytical DDM 2910 Automatic Density Meter. Once calibrated by checking the value of air density at $293.15 \mathrm{~K}, 2 \mathrm{~mL}$ of each sample were withdrawn into a syringe and then released in a U-tube until filled. Careful rinsing with ethanol was operated between each measurement. The density measurements were done between 293.15 and $323.15 \mathrm{~K}$ every $10 \mathrm{~K}$. In this temperature range, an absolute average deviation $\mathrm{AAD}=0.15 \%$ was obtained between experimental data of distilled water and reference data from ref. [16]. Also, the absolute average deviation AAD is $0.046 \%$ with Tyfocor ${ }^{\circledR}$ LS in comparison to available manufacturer data [17].

The temperature range considered here is linked to the available temperatures of the density measurement apparatus. Consequently, to extend the range of density values for all samples to lower and higher temperatures (from $273.15-283.15$ to $333.15 \mathrm{~K}$ ) than those experimentally measured (from 293.15 to $323.15 \mathrm{~K}$ ), and also to evaluate ST at these temperatures, the experimental data of density were correlated to equation (1) $[18,19]$.

$$
\rho(T)=A_{0}+A_{1} T+A_{2} T^{2}
$$

Where $\rho$ is the density in $\mathrm{kg} \cdot \mathrm{m}^{-3}$ and $\mathrm{T}$ is the temperature in $\mathrm{K}$. The coefficients $\mathrm{A}_{\mathrm{i}}$ of each sample are gathered in Table 2 along with the respective standard deviation (lower than 0.025 $\left.\mathrm{kg} \cdot \mathrm{m}^{-3}\right)$.

Finally, ST measurements were performed with a Drop Shape Analyzer DSA-30 from KRÜSS GmBH (Hamburg, Germany) based on the pendant drop technique. Typically, a 15-gauge needle with an outer diameter of $1.835 \mathrm{~mm}$ was used to produce drops at controlled flow rate and volume within a temperature chamber. The temperature in the chamber was regulated with a 
Peltier system and controlled by a PT100 probe. The comprehensive experimental procedure was similar to the one described in ref. [20]. In the pendant drop configuration, Young-Laplace equation was used for determining ST from the image analysis of the drop shape produced at the end of the needle and the density of the sample. Each ST values reported in the following is the average of at least 10 measurements. ST of DW was measured in the temperature range 283.15333.15 K (see later in Figure 5). An absolute average deviation AAD $=1.08 \%$ was obtained between experimental data and reference data in this temperature range [21].

\section{Results and discussion}

\subsection{Chemical modification of MWCNTs and dispersion in the base fluids}

Purity of the treated MWCNTs was quantified by TGA (Figure 1a). The thermogram of the raw MWCNTs shows one weight loss at around $773.15 \mathrm{~K}$ assigned to the combustion of carbon species which represents 88 wt.\% of the sample and a remaining metallic impurity rate of 12 wt.\% in their oxide forms (i.e. $\mathrm{Fe}_{2} \mathrm{O}_{3}, \mathrm{CoO}, \mathrm{Al}_{2} \mathrm{O}_{3}$ ). After the purification treatment, the remaining weight being close to zero, the sample was completely free from metal and the combustion temperature of MWCNTs is shifted to higher temperatures (+ $100 \mathrm{~K})$. As expected, the purified MWCNTs are more stable against combustion than the raw MWCNTs; metallic impurities playing the role of combustion catalyst of CNTs [22]. Moreover, no mineral impurity pollutes the MWCNTs after functionalization (100 wt.\% loss after MWCNT combustion) as it can be sometimes observed after using a $\mathrm{KMnO}_{4}$-based treatment [23].

Figure $1 \mathrm{~b}$ shows the weight losses from TGA in helium of the raw, purified and the two functionalized MWCNT samples. Compared to the raw and the purified samples, the functionalized MWCNTs show a more pronounced weight loss attributed to the functional group 
release. The purified MWCNT sample loses only 1.49 wt.\% of its mass in agreement with the weak amount of native functional groups on the raw MWCNT walls. The functionalized MWCNTs show a weight loss of 13.37 wt.\% (at $1173.15 \mathrm{~K}$ ).
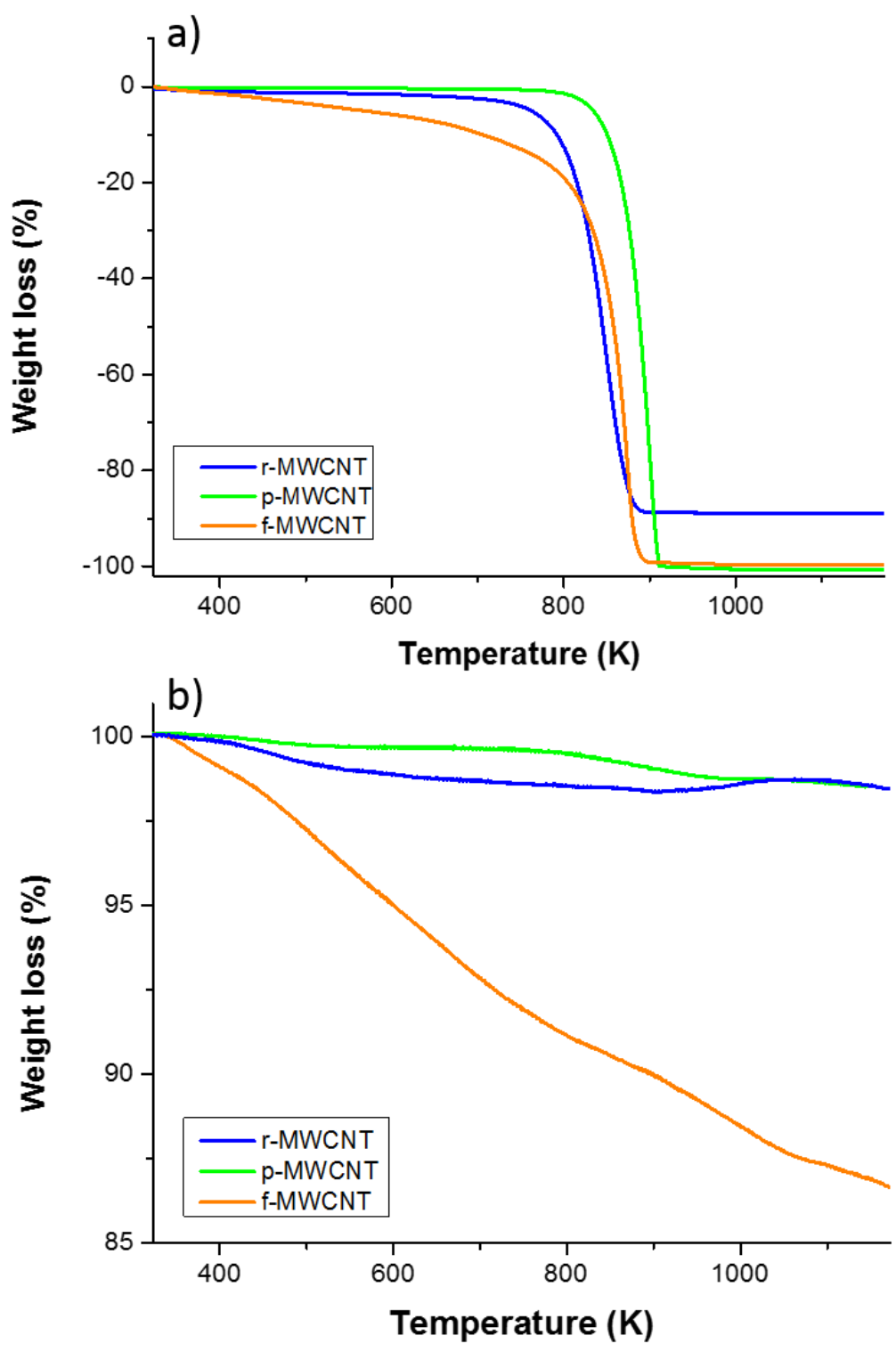
Figure 1. Thermograms recorded of r-MWCNT (blue curve), p-MWCNT (green curve), fMWCNT (orange curve); a) in dry air and b) in helium.

Figure 2 shows typical TEM images of the MWCNTs before (a and d) and after the two chemical treatments: purification ( $b$ and e) and functionalization ( $c$ and $f$ ) with different magnifications. In the raw MWCNTs, the metal impurity particles (dark particles top right Fig. 2a) are clearly identified and they are no more present is the purified (Fig. 2b) and in the functionalized (Fig. 2c) sample in agreement with the TGA results (Fig. 1a). In higher magnification images, while no damaging on the nanotubes is observable after purification (Fig. 2e), the external MWCNT walls were slightly attacked by the functionalization leaving however untouched the internal walls (Fig. 2f).
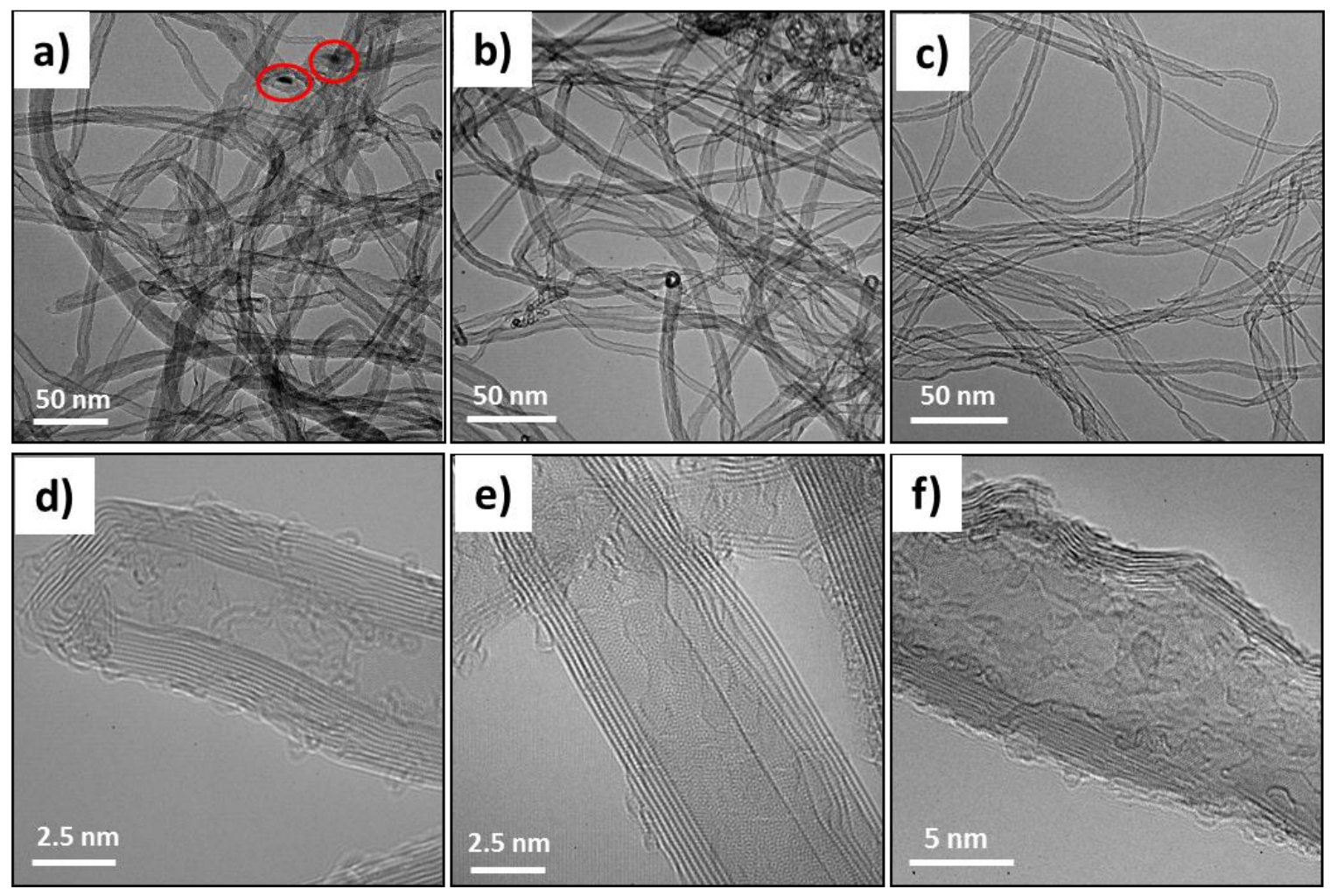
Figure 2. Typical TEM bright field micrographs of the MWCNT samples. a) and d) r-MWCNT; b) and e) p-MWCNT; c) and f) f-MWCNT at different magnifications.

Raman spectroscopy is a well suitable technique for the structural quality assessment of CNTs because it is very sensitive to the evolution of the sample according to chemical treatments [24]. The intensity of the D band (around $1350 \mathrm{~cm}^{-1}$ ) is sensitive to any introduction of defects, for example $\mathrm{sp}^{2}$ carbon atoms turning $\mathrm{sp}^{3}$ hybridization through functionalization. Meanwhile, the $\mathrm{G}$ band (around $1590 \mathrm{~cm}^{-1}$ ) is representative of the graphitic carbon $\mathrm{sp}^{2}$ network. Compared to the raw MWCNTs $\left(\mathrm{I}_{\mathrm{D}} / \mathrm{I}_{\mathrm{G}}=1.87 \pm 0.04\right)$, the purified MWCNTs show a decrease of the $\mathrm{I}_{\mathrm{D}} / \mathrm{I}_{\mathrm{G}}(1.59 \pm$ 0.08) meaning that no additional defect has been introduced during our purification treatment. $\mathrm{I}_{\mathrm{D}} / \mathrm{I}_{\mathrm{G}}$ is increased for $\mathrm{f}-\mathrm{MWCNT}$ up to $\mathrm{I}_{\mathrm{D}} / \mathrm{I}_{\mathrm{G}}=2.32 \pm 0.01 . \mathrm{I}_{\mathrm{D}} / \mathrm{I}_{\mathrm{G}}$ is thus significantly increased from 1.59 to 2.32 through functionalization giving a clear evidence of presence of functional groups at the MWCNT surface.

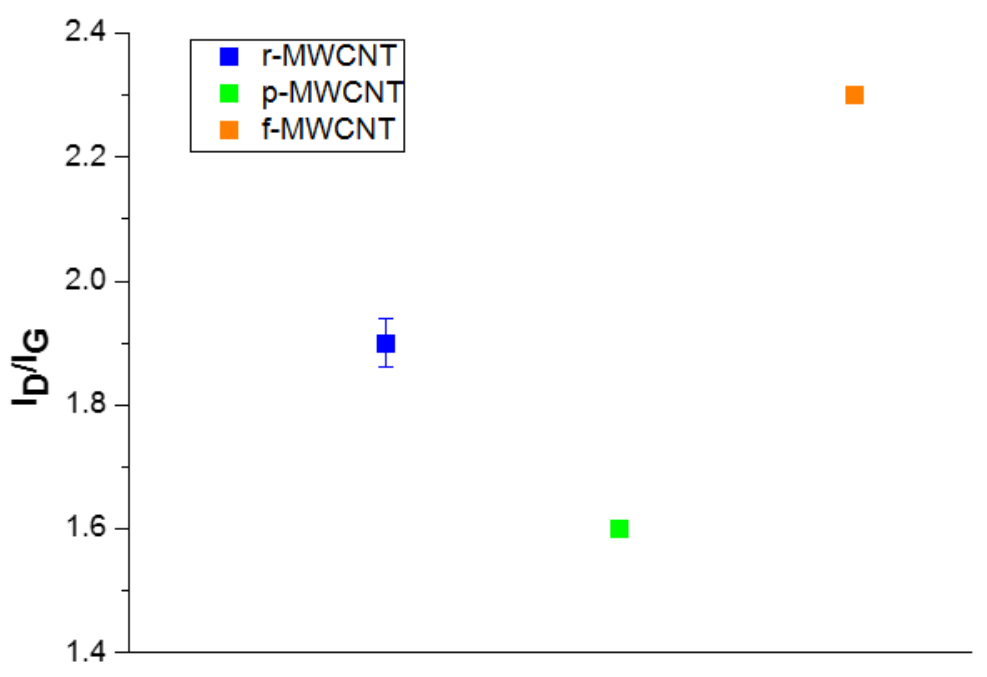


Figure 3. $I_{D} / I_{G}$ ratios of r-MWCNT (blue), p-MWCNT (green) and f-MWCNT (orange). The error bars were determined from the standard deviation to the calculated ID/IG average.

By assuming that the mass loss of the functionalized MWCNTs at $1173.15 \mathrm{~K}$ from TGA (Fig. 1) is due to release of mainly carboxyl acids as expected by the used functionalization method [15], the functionalization level can be estimated from the following relation equation(2) [25]:

$$
n=\frac{M_{\mathrm{COOH}}}{M_{C}}\left(\frac{100}{W L}-1\right)
$$

Where $\mathrm{M}_{\mathrm{COOH}}$ and $\mathrm{M}_{\mathrm{C}}$ are the respective molar weights of a carboxyl acid group and a carbon atom; and WL, the weight assigned to the functional group loss from TGA. The weight loss at 1173.15 $\mathrm{K}$ is of about 1.49 and $13.37 \mathrm{wt} \%$ for the MWCNTs before and after the chemical treatment, respectively. For f-MWCNT, the weight loss due to detachment of carboxyl groups is of 11.88 wt.\%. $\mathrm{n}$ determined from (1) is about 28 . That means that 1 functional group is grafted every 28 carbon atoms or every almost $5 \mathrm{C}_{6}$-rings, in average. The found functionalization level is then relatively low. This seems to indicate that hydrophobic domains are probably still present on the MWCNT surface. Indeed, even if the internal walls remain untouched after the applied chemical treatment, this kind of applied oxidation is known to preferentially occur at the defect sites and the extremities of the MWCNTs leading to a non-homogeneous positioning of the functional groups on the MWCNT surface [26,27]. At the nanometer scale, some areas (cylindrical parts of nanotubes) are certainly more hydrophobic and other areas (MWCNT extremities) more hydrophilic; these latter playing the major role for the CNT dispersion within both DW and Tyfocor. 
Optical microscopy images shown in Figure 4 evidence that both prepared nanofluids (in DW, Fig. 4a and Tyfocor, Fig. 4b) with 0.1 wt.\% of f-MWCNT appear very homogeneous. Only very few aggregates are visible in the water-based nanofluid. Presence of propylene glycol in Tyfocor seems to improve the MWCNT dispersion. These images are in agreement with the visual observations of the nanofluid sample in vials. While the functionalization level is low, the number of functional groups present on the f-MWCNT walls is high enough to counterbalance the van der Waals interactions between the MWCNTs, leading to a good quality of dispersion. This agrees well also with the value of the zeta potential of f-MWCNT in DW measured at - 42 $\mathrm{mV}$ indicating a good dispersion and stability [28,29]. It should be mentioned that zeta potential distribution for dispersion in Tyfocor is too large certainly due to the presence of the charged additives in this commercial mixture leading to a non-reliable value.

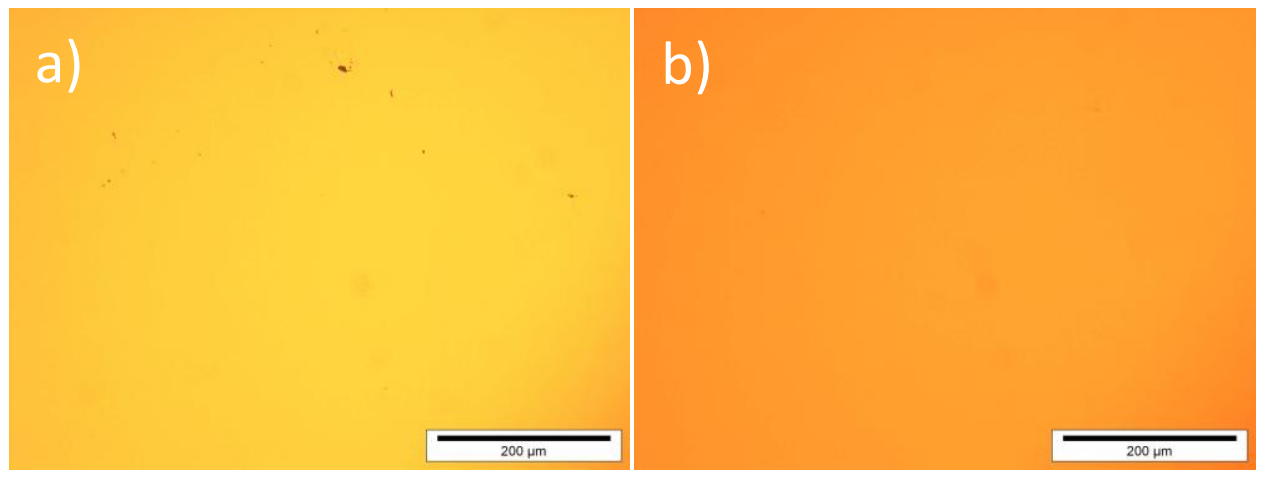

Figure 4. Optical microscope images of dispersed f-MWCNT $(0.1$ wt.\%) in DW (a) and in Tyfocor (b).

\subsection{Density of base fluids and nanofluids}

The experimental density values of the used base fluids and nanofluids are gathered in Table 1. In this Table are also reported the computed density values obtained from equation (1) for 
temperatures lower than $273.15 \mathrm{~K}$ and higher than $323.15 \mathrm{~K}$. As mentioned previously, the coefficients $A_{i}$ for each sample and the respective standard deviations from equation (1) in the temperature range $273.15-323.15 \mathrm{~K}$ are included in Table 2 . The density of both base fluids and the corresponding nanofluids decreases as the temperature increases with a similar trend; the Tyfocor density being higher than that of water due to the presence of propylene-glycol. Moreover, the density of nanofluids with the lowest content in MWCNTs $(0.001 \mathrm{wt} \%)$ is logically very close (almost similar) to that of the respective base fluid by taking into account the measurement uncertainty. For both base fluids, it is observed that the density for $0.1 \mathrm{wt} . \%$ of MWCNTs is slightly higher than that of the respective base fluid. An average increase in density of $0.05 \%$ in the temperature range $273.15-323.15 \mathrm{~K}$ is noticed for the water-based nanofluid. With Tyfocor as base fluid, the average rise of density in the same temperature range is around $0.08 \%$.

Table 1. Experimental densities and computed densities (in bold) from equation (1) of the used base fluids and the nanofluids with different MWCNT mass fractions and temperatures

\begin{tabular}{|c|c|c|c|c|c|c|}
\hline & \multicolumn{3}{|c|}{ Water as base fluid } & \multicolumn{3}{c|}{ Tyfocor as base fluid } \\
\hline MWCNT mass content / wt\% & 0 & 0.001 & 0.1 & 0 & 0.001 & 0.1 \\
\hline T/K & \multicolumn{7}{|c|}{$\rho / \mathrm{kg} . \mathrm{m}^{-3}$} \\
\hline 273.15 & - & - & - & $\mathbf{1 0 4 5 . 4 0 5}$ & $\mathbf{1 0 4 5 . 4 0 5}$ & $\mathbf{1 0 4 6 . 3 9 5}$ \\
\hline 283.15 & $\mathbf{9 9 9 . 9 7 5}$ & $\mathbf{9 9 9 . 9 7 5}$ & $\mathbf{1 0 0 0 . 6}$ & $\mathbf{1 0 4 0 . 0 2 5}$ & $\mathbf{1 0 4 0 . 0 2 5}$ & $\mathbf{1 0 4 0 . 9 7 5}$ \\
\hline 293.15 & 998.1 & 998.1 & 998.6 & 1034.3 & 1034.3 & 1035.2 \\
\hline 303.15 & 995.5 & 995.5 & 996 & 1028.2 & 1028.2 & 1029.1 \\
\hline 313.15 & 992.1 & 992.1 & 992.5 & 1021.8 & 1021.8 & 1022.6 \\
\hline 323.15 & 988.1 & 988.1 & 988.5 & 1015.1 & 1015.1 & 1015.8 \\
\hline 333.15 & $\mathbf{9 8 3 . 1 2 5}$ & $\mathbf{9 8 3 . 1 2 5}$ & $\mathbf{9 8 3 . 7}$ & $\mathbf{1 0 0 7 . 8 7 5}$ & $\mathbf{1 0 0 7 . 8 7 5}$ & $\mathbf{1 0 0 8 . 6 2 5}$ \\
\hline
\end{tabular}

Table 2. Fitting parameters $A_{i}$ and standard deviations (St. Dev.) from equation (1) for the base fluids and nanofluids at different mass fraction

\begin{tabular}{|c|c|c|c|c|c|c|}
\hline & \multicolumn{3}{|c|}{ Water as base fluid } & \multicolumn{3}{c|}{ Tyfocor as base fluid } \\
\hline $\begin{array}{c}\text { MWCNT mass } \\
\text { content / wt.\% }\end{array}$ & 0 & 0.001 & 0.1 & 0 & 0.001 & 0.1 \\
\hline $\mathrm{A}_{0} / \mathrm{kg} \cdot \mathrm{m}^{-3}$ & 741.6537156 & 741.653716 & 766.1447213 & 1057.01046 & 1057.01046 & 1059.09306 \\
\hline $\mathrm{A}_{1} / \mathrm{kg} \cdot \mathrm{m}^{-3} \cdot \mathrm{K}^{-1}$ & 1.974125 & 1.974125 & 1.81905 & 0.435525 & 0.435525 & 0.431525 \\
\hline
\end{tabular}




\begin{tabular}{|c|c|c|c|c|c|c|}
\hline $\mathrm{A}_{2} / \mathrm{kg} \cdot \mathrm{m}^{-3} \cdot \mathrm{K}^{-2}$ & -0.00375 & -0.00375 & -0.0035 & -0.00175 & -0.00175 & -0.00175 \\
\hline St. Dev. / kg.m & 0.022361 & 0.022362 & 0.024443 & 0.022361 & 0.022361 & 0.022363 \\
\hline $\begin{array}{c}\text { Regression } \\
\text { Coefficient } \mathrm{R}^{2}\end{array}$ & 0.999995641 & 0.99999564 & 0.999930577 & 0.99999879 & 0.99999879 & 0.99999881 \\
\hline
\end{tabular}

\subsection{Surface tension of base fluids and nanofluids}

Measurements of ST of base fluids and nanofluids at two weight concentrations 0.001 and 0.1 wt.\%, for a temperature range of 283.15-333.15 K for the DW-based nanofluids and 273.15$333.15 \mathrm{~K}$ for the Tyfocor-based nanofluids, are reported in figures 5 and 6 , respectively. The error bars were determined from the standard deviation to the average value of ST.

First at all, it is observed in Figure 5 that ST of DW decreases with temperature, as expected, and the found values are in good agreement with literature data [21] as explained in section 2. As shown in Figure 6, ST of Tyfocor is lower than that of DW due to the presence of propylene glycol. Tyfocor ST decreases as well with temperature. No comparison can be made with literature data since this property is reported for the first time with this commercial heat transfer fluid. It is also observed from both figures that reduction rate in ST with temperature is greater for DW. While the densities of nanofluids slightly increases with nanotubes content in comparison to the density of base fluids, as shown before in section 3.2, it appears that ST of nanofluids evolves in different manner following the base fluid and changes are more pronounced, as explained below.

Figure 5 also shows the evolution of ST as a function of temperature and concentration for $\mathrm{f}$ MWCNT/DW-based nanofluids. The ST of these nanofluids is lowered in average by 1.83 and $3.96 \%$ as the concentration of $\mathrm{f}-\mathrm{MWCNT}$ increases from 0.001 to $0.1 \mathrm{wt} . \%$, respectively. The observed trend is consistent with results previously reported in [5], as well as with other carbon structures such as graphene oxide and reduced graphene oxide dispersed in water [30]. As 
previously mentioned, f-MWCNT is well dispersed in DW due to the grafted functional groups and the functionalized MWCNTs also bear hydrophobic zones which affinity with air/water interface is expected to increase, leading to the observed ST reduction with MWCNT concentration. For the nanofluids prepared with Tyfocor as base fluid, contrary to what is it observed with DW, increase in f-MWCNT concentration induces an increase in ST: ST is of 7.48 and $10.41 \%$ at $273.15 \mathrm{~K}$ and 8.87 and $19.43 \%$ at $333.15 \mathrm{~K}$ for MWCNT concentration of 0.001 and $0.1 \mathrm{wt} . \%$, respectively (Fig. 6). We hypothesize that covering of the remaining hydrophobic surface of MWCNTs by the polymer chains of propylene glycol could increase affinity between MWCNTs and Tyfocor leading to the observed increase in ST [31,32]. Indeed, alcohols are well known to play the role of surfactants or co-surfactants, useful to help hydrophobic nanoparticles or colloids to be dispersed in water [33]. Affinity of f-MWCNTs with Tyfocor is consequently better with DW in agreement with optical observations (Fig. 4). For Tyfocor-based nanofluids, fMWCNT understandably remain within the bulk liquid, and only a few are transported to the interfacial region due to Brownian motion. The attraction between the nanotubes and propylene glycol contained in Tyfocor reduces the intermolecular spacing resulting in an increase of the ST. This also leads to a raise in ST when the MWCNT content increases. Finally, as reported in literature [2], the ST of all the prepared nanofluids decreases with the raise of temperature whatever the base fluid used. 


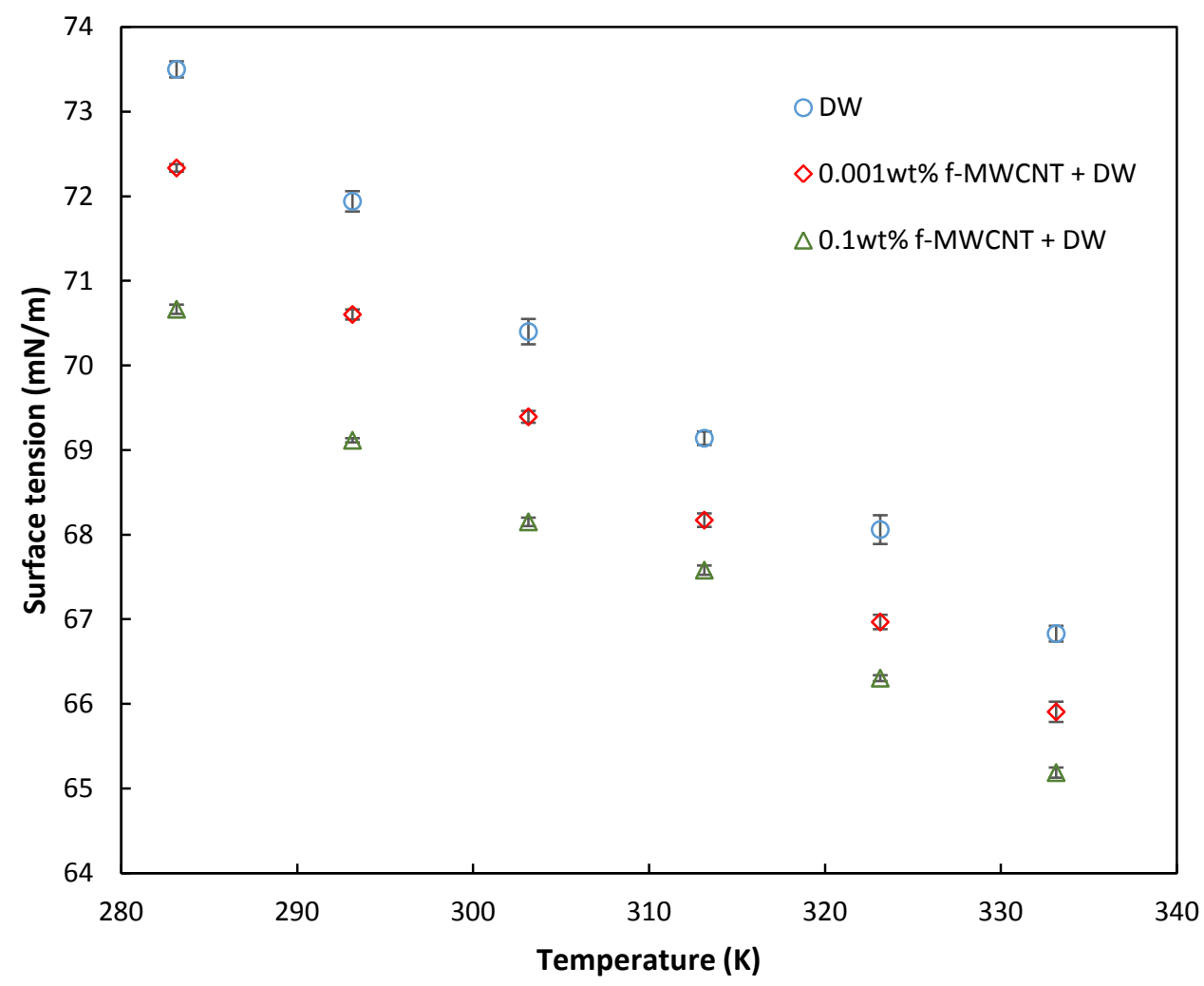

Figure 5. Surface tension of the f-MWCNT nanofluids with DW as base fluid - influence of concentration and temperature. 


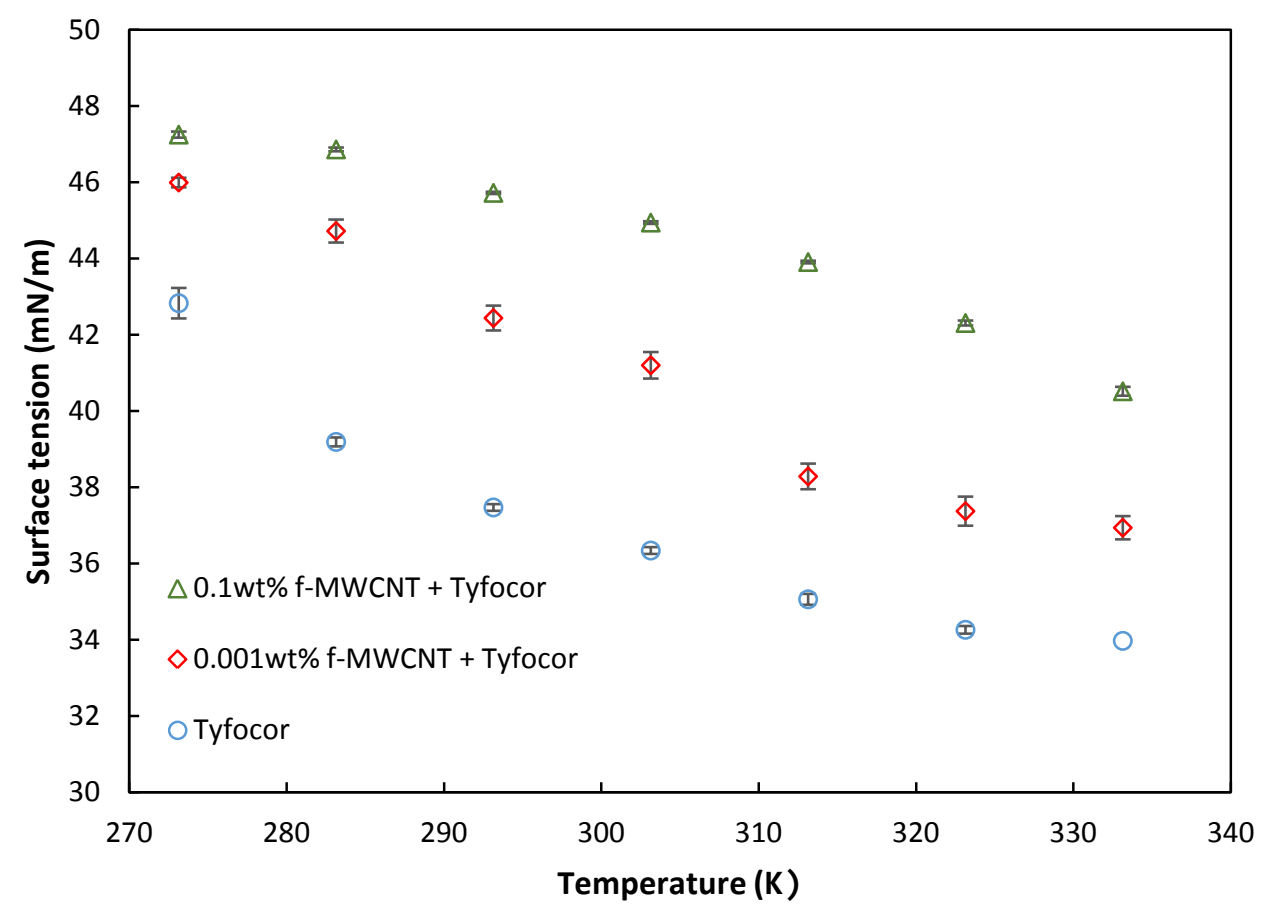

Figure 6. Surface tension of the prepared f-MWCNT nanofluids with Tyfocor as base fluid - Influence of concentration and temperature.

\section{Conclusions}

In this study, MWCNT-based nanofluids were prepared with distilled water and propyleneglycol/water (Tyfocor) without using surfactant. The MWCNTs were chemically oxidized with $\mathrm{KMnO}_{4}$. A comprehensive characterization of the raw and the chemically modified MWCNTs by using thermogravimetric analysis (TGA), transmission electron microscopy (TEM) and Raman spectroscopy was performed evidencing the achievement of the functionalization process. The used MWCNT concentration were 0.001 and $0.1 \mathrm{wt} . \%$. The densities of nanofluids are slightly modified by the MWCNT concentration in comparison to the densities of base fluids. Behavior of the ST of the nanofluids measured by the pendant drop method was analyzed and discussed as a function of the MWCNT weight loading, temperature and the base fluid used. To 
the best of our knowledge, the ST of MWCNT/Tyfocor-based nanofluids was investigated for the first time in the present study. As expected, a decrease in ST with temperature was observed for both kinds of nanofluids. Our results show an opposite tendency regarding ST with MWCNT concentration for the two used base fluids: a reduction in ST was obtained with distilled water as base fluid and a significant increase in ST was observed with Tyfocor. This phenomenon was explained by the surface properties and the possible induced interactions within each nanofluid, i.e. functionalized MWCNT/distilled water and functionalized MWCNT/Tyfocor nanofluids.

\section{Conflicts of interest}

There are no conflicts to declare

\section{Aknowledgements:}

The authors thank Mr. P. Franchetti and Mr. L. Aranda for their help for TGA and Raman spectroscopy experiments, respectively. The authors are grateful to Dr. F. Michaux for his help for zeta potential measurements. The authors thank the University of Lorraine for its financial support especially for Miss. N. Berrada’s Doctoral Fellowship. Dr. Patrice Estellé acknowledges the European Union through the European Regional Development Fund (ERDF), the Ministry of Higher Education and Research, the French region of Brittany and Rennes Métropole for the financial support of surface tension experimental device.

\section{References}

[1] O. Mahian, L. Kolsi, M. Amani, P. Estelle, G. Ahmadi, C. Kleinstreuer, J.S. Marshalli, M. Siavashi, R.A. Taylor, H. Niazmand, S. Wongwises, T. Hayat, A. Kolanjiyil, A. Kasaeian, L. Pop, Recent advances in 
modeling and simulation of nanofluid flows-Part I: Fundamentals and theory, Phys. Rep.-Rev. Sec. Phys. Lett. 790 (2019) 1-48. doi:10.1016/j.physrep.2018.11.004.

[2] P. Estelle, D. Cabaleiro, G. Zyla, L. Lugo, S.M.S. Murshed, Current trends in surface tension and wetting behavior of nanofluids, Renew. Sust. Energ. Rev. 94 (2018) 931-944. doi:10.1016/j.rser.2018.07.006.

[3] M. Hernaiz, V. Alonso, P. Estelle, Z. Wu, B. Sunden, L. Doretti, S. Mancin, N. Cobanoglu, Z.H. Karadeniz, N. Garmendia, M. Lasheras-Zubiate, L. Hernandez Lopez, R. Mondragon, R. MartinezCuenca, S. Barison, A. Kujawska, A. Turgut, A. Amigo, G. Huminic, A. Huminic, M.-R. Kalus, K.-G. Schroth, M.H. Buschmann, The contact angle of nanofluids as thermophysical property, J. Colloid Interface Sci. 547 (2019) 393-406. doi:10.1016/j.jcis.2019.04.007.

[4] H.S. Xue, J.R. Fan, Y.C. Hu, R.H. Hong, K.F. Cen, The interface effect of carbon nanotube suspension on the thermal performance of a two-phase closed thermosyphon, J. Appl. Phys. 100 (2006) 104909. doi:10.1063/1.2357705.

[5] S. Tanvir, L. Qiao, Surface tension of Nanofluid-type fuels containing suspended nanomaterials, Nanoscale Research Letters. 7 (2012) 226. doi:10.1186/1556-276X-7-226.

[6] S.M.S. Murshed, D. Milanova, R. Kumar, An Experimental Study of Surface Tension-Dependent Pool Boiling Characteristics of Carbon Nanotubes-Nanofluids, Amer Soc Mechanical Engineers, New York, 2009.

[7] R. Kumar, D. Milanova, Effect of surface tension on nanotube nanofluids, Appl. Phys. Lett. 94 (2009) 073107. doi:10.1063/1.3085766.

[8] A. Karthikeyan, S. Coulombe, A.M. Kietzig, Wetting behavior of multi-walled carbon nanotube nanofluids, Nanotechnology. 28 (2017) 105706. doi:10.1088/1361-6528/aa5a5f.

[9] H. Soleimani, M.K. Baig, N. Yahya, L. Khodapanah, M. Sabet, B.M.R. Demiral, M. Burda, Impact of carbon nanotubes based nanofluid on oil recovery efficiency using core flooding, Results Phys. 9 (2018) 39-48. doi:10.1016/j.rinp.2018.01.072.

[10] M. Ershadi, M. Alaei, A. Rashidi, A. Ramazani, S. Khosravani, Carbonate and sandstone reservoirs wettability improvement without using surfactants for Chemical Enhanced Oil Recovery (C-EOR), Fuel. 153 (2015) 408-415. doi:10.1016/j.fuel.2015.02.060.

[11] M. AfzaliTabar, M. Alaei, R.R. Khojasteh, F. Motiee, A.M. Rashidi, Preference of multi-walled carbon nanotube (MWCNT) to single-walled carbon nanotube (SWCNT) and activated carbon for preparing silica nanohybrid pickering emulsion for chemical enhanced oil recovery (C-EOR), J. Solid State Chem. 245 (2017) 164-173. doi:10.1016/j.jssc.2016.10.017.

[12] M.A. Oyinlola, G.S.F. Shire, R.W. Moss, Thermal analysis of a solar collector absorber plate with microchannels, Exp. Therm. Fluid Sci. 67 (2015) 102-109. doi:10.1016/j.expthermflusci.2014.10.014.

[13] P. Estelle, S. Halelfadl, T. Mare, Thermophysical properties and heat transfer performance of carbon nanotubes water-based nanofluids, J. Therm. Anal. Calorim. 127 (2017) 2075-2081. doi:10.1007/s10973-016-5833-8.

[14] G. Mercier, C. Herold, J.-F. Mareche, S. Cahen, J. Gleize, J. Ghanbaja, G. Lamura, C. Bellouard, B. Vigolo, Selective removal of metal impurities from single walled carbon nanotube samples, New J. Chem. 37 (2013) 790-795. doi:10.1039/c2nj41057e.

[15] J. Zhang, H.L. Zou, Q. Qing, Y.L. Yang, Q.W. Li, Z.F. Liu, X.Y. Guo, Z.L. Du, Effect of chemical oxidation on the structure of single-walled carbon nanotubes, J. Phys. Chem. B. 107 (2003) 3712-3718. doi:10.1021/jp027500u.

[16] J. Sengers, J. Watson, Improved International Formulations for the Viscosity and ThermalConductivity of Water Substance, J. Phys. Chem. Ref. Data. 15 (1986) 1291-1314. doi:10.1063/1.555763.

[17] Tyfocor LS Technical Information available at https://tyfo.de/en/produkt/tyfocor-ls/, (n.d.). 
[18] D. Cabaleiro, M.J. Pastoriza-Gallego, M.M. Pineiro, J.L. Legido, L. Lugo, Thermophysical properties of (diphenyl ether plus biphenyl) mixtures for their use as heat transfer fluids, J. Chem. Thermodyn. 50 (2012) 80-88. doi:10.1016/j.jct.2012.02.001.

[19] J.P. Vallejo, J. Perez-Tavernier, D. Cabaleiro, J. Fernandez-Seara, L. Lugo, Potential heat transfer enhancement of functionalized graphene nanoplatelet dispersions in a propylene glycol-water mixture. Thermophysical profile, J. Chem. Thermodyn. 123 (2018) 174-184. doi:10.1016/j.jct.2018.04.007.

[20] R. Gomez-Villarejo, T. Aguilar, S. Hamze, P. Estelle, J. Navas, Experimental analysis of water-based nanofluids using boron nitride nanotubes with improved thermal properties, J. Mol. Liq. 277 (2019) 93-103. doi:10.1016/j.molliq.2018.12.093.

[21] N. Vargaftik, B. Volkov, L. Voljak, International Tables of the Surface-Tension of Water, J. Phys. Chem. Ref. Data. 12 (1983) 817-820. doi:10.1063/1.555688.

[22] I.W. Chiang, B.E. Brinson, R.E. Smalley, J.L. Margrave, R.H. Hauge, Purification and characterization of single-wall carbon nanotubes, J. Phys. Chem. B. 105 (2001) 1157-1161. doi:10.1021/jp003453z.

[23] N.Y. Zhang, J. Me, V.K. Varadan, Functionalization of carbon nanotubes by potassium permanganate assisted with phase transfer catalyst, Smart Mater. Struct. 11 (2002) 962-965. doi:10.1088/0964$1726 / 11 / 6 / 318$.

[24] S. Osswald, M. Havel, Y. Gogotsi, Monitoring oxidation of multiwalled carbon nanotubes by Raman spectroscopy, J. Raman Spectrosc. 38 (2007) 728-736. doi:10.1002/jrs.1686.

[25] V. Mamane, G. Mercier, J.A. Shukor, J. Gleize, A. Azizan, Y. Fort, B. Vigolo, Chemi- vs physisorption in the radical functionalization of single-walled carbon nanotubes under microwaves, Beilstein $\mathrm{J}$. Nanotechnol. 5 (2014) 537-545. doi:10.3762/bjnano.5.63.

[26]X. Devaux, B. Vigolo, E. McRae, F. Valsaque, N. Allali, V. Mamane, Y. Fort, A.V. Soldatov, M. Dossot, S.Y. Tsareva, Covalent Functionalization of HiPco Single-Walled Carbon Nanotubes: Differences in the Oxidizing Action of $\mathrm{H} 2 \mathrm{SO} 4$ and $\mathrm{HNO} 3$ during a Soft Oxidation Process, ChemPhysChem. 16 (2015) 2692-2701. doi:10.1002/cphc.201500248.

[27] A. Hirsch, Functionalization of single-walled carbon nanotubes, Angew. Chem.-Int. Edit. 41 (2002) 1853-1859. doi:10.1002/1521-3773(20020603)41:11<1853::AID-ANIE1853>3.0.CO;2-N.

[28] A. Ghadimi, R. Saidur, H.S.C. Metselaar, A review of nanofluid stability properties and characterization in stationary conditions, International Journal of Heat and Mass Transfer. 54 (2011) 4051-4068. doi:10.1016/j.ijheatmasstransfer.2011.04.014.

[29] J.-H. Lee, K.S. Hwang, S.P. Jang, B.H. Lee, J.H. Kim, S.U.S. Choi, C.J. Choi, Effective viscosities and thermal conductivities of aqueous nanofluids containing of Al2O3 low volume concentrations nanoparticles, Int. J. Heat Mass Transf. 51 (2008) 2651-2656. doi:10.1016/j.ijheatmasstransfer.2007.10.026.

[30] D. Cabaleiro, P. Estelle, H. Navas, A. Desforges, B. Vigolo, Dynamic Viscosity and Surface Tension of Stable Graphene Oxide and Reduced Graphene Oxide Aqueous Nanofluids, J. Nanofluids. 7 (2018) 1081-1088. doi:10.1166/jon.2018.1539.

[31] K. Balamurugan, P. Baskar, R. Mahesh Kumar, S. Das, V. Subramanian, Interaction of Carbon Nanotube with Ethylene Glycol-Water Binary Mixture: A Molecular Dynamics and Density Functional Theory Investigation, J. Phys. Chem. C. 116 (2012) 4365-4373. doi:10.1021/jp206882f.

[32] A.H. Mary, K.S. Suganthi, K.S. Rajan, Mechanistic Investigations of Viscosity and Thermal Conductivity Enhancement in Multi-Walled Carbon Nanotubes-Propylene Glycol Nanofluids, Nanosci. Nanotechnol. Lett. 5 (2013) 1125-1129. doi:10.1166/nnl.2013.1673.

[33] I. Kralova, J. Sjoblom, Surfactants Used in Food Industry: A Review, J. Dispersion Sci. Technol. 30 (2009) 1363-1383. doi:10.1080/01932690902735561. 